\title{
Highlights from SuperKEKB Commissioning for Early Stage of Nano-Beam Scheme and Crab Waist Scheme
}

\author{
Y. Ohnishi ${ }^{\dagger}, *$ \\ High Energy Accelerator Research Organization (KEK), \\ Oho 1-1, Tsukuba, Japan \\ E-mail: yukiyoshi.onishi@kek.jp
}

The SuperKEKB electron-positron collider is being commissioned at KEK to study new physics in the B-meson decays. In order to accomplish this purpose, the luminosity of 40 times of the highest luminosity record at KEKB, $8 \times 10^{35} \mathrm{~cm}^{-2} \mathrm{~s}^{-1}$ is necessary. We have applied a novel "nano-beam scheme" to squeeze the beta function at the interaction point (IP) down to $1 \mathrm{~mm}$ in the vertical, 60 $\mathrm{mm}$ for the HER ( $7 \mathrm{GeV}$ electrons) and $80 \mathrm{~mm}$ for the LER (4 GeV positrons) in the horizontal direction, respectively. We have tested $\beta_{y}^{*}$ of $800 \mu \mathrm{m}$ finally and performed physics run with data acquisitions by the Belle II detector. The beta function at the IP is the smallest value for the existing circular colliders in the world. However, the design value is $0.3 \mathrm{~mm}$ which is still about $1 / 3$ of the achievement. We have also applied a "crab waist scheme" proposed by P. Raimondi et al. to improve the luminosity performance in the nano-beam scheme. The peak luminosity of $2.4 \times 10^{34} \mathrm{~cm}^{-2} \mathrm{~s}^{-1}$ has been achieved which is the highest value in the world. The vertical beam size at the IP of $224 \mu \mathrm{m}$ was also achieved, which is the smallest beam size for the colliders. The early stage of the commissioning of the nano-beam scheme as well as the crab waist scheme in 2019 run and Spring run in 2020 is presented.

40th International Conference on High Energy physics - ICHEP2020

July 28 - August 6, 2020

Prague, Czech Republic (virtual meeting)

\footnotetext{
*Speaker

$\dagger$ on behalf of the SuperKEKB accelerator group
} 


\section{Nano-beam scheme}

The luminosity is determined by the beam currents, the vertical beta function at the $\operatorname{IP}\left(\beta_{y}^{*}\right)$, and the limit of vertical beam-beam interaction $\left(\xi_{y}\right)$ in principle. The benefit of the smaller $\beta_{y}^{*}$ than the bunch length $\left(\sigma_{z}\right)$ is less obtained for the previous colliders before SuperKEKB [1]. The deterioration of luminosity comes from the geometrical loss which is called an "hourglas" effect. However, the nano-beam scheme overcomes this difficulty by using a small emittance with a large Piwinski angle. The luminosity formula in the nano-beam scheme can be described by

$$
L=\frac{N_{+} N_{-} n_{b} f_{0}}{2 \pi \Sigma_{x}^{*} \Sigma_{y}^{*}}=\frac{N_{+} N_{-} n_{b} f_{0}}{2 \pi \phi_{x} \sqrt{\sigma_{z+}^{2}+\sigma_{z-}^{2}} \sqrt{\varepsilon_{y+} \beta_{y+}^{*}+\varepsilon_{y-} \beta_{y-}^{*}}},
$$

where $N_{ \pm}$is a particle population in a bunch, $n_{b}$ is a number of bunches, and $f_{0}$ is a revolution frequency. The variable at the IP is denoted by $*$. The $\Sigma_{x}^{*}$ can be written with a half crossing angle, $\phi_{x}$, by

$$
\Sigma_{x}^{*}=\sqrt{\sigma_{x+}^{* 2}+\sigma_{z+}^{2} \tan ^{2} \phi_{x}+\sigma_{x-}^{* 2}+\sigma_{z-}^{2} \tan ^{2} \phi_{x}}=\sqrt{\sigma_{x+}^{* 2}\left(1+\Psi_{+}^{2}\right)+\sigma_{x-}^{* 2}\left(1+\Psi_{-}^{2}\right)},
$$

where the Piwinski angle is

$$
\Psi_{ \pm}=\frac{\sigma_{z \pm}}{\sigma_{x \pm}^{*}} \tan \phi_{x}
$$

In the case of an ordinary collision scheme such as a head-on collision and a small crossing-angle, $\Psi_{ \pm}$is much smaller than 1 . The nano-beam scheme applies a large Piwinski angle larger than $\mathrm{O}(10)$, then the luminosity formula becomes Eq. (1). The real horizontal beam size at the IP $\left(\sigma_{x}^{*}\right)$ is not present in the formula, however, the effective horizontal beam size can be large. Consequently, the luminosity decreases geometrically. In order to compensate the geometrical luminosity loss due to the effective horizontal beam size, the vertical emittance $\left(\varepsilon_{y}\right)$ is required to be small enough.

Figure 1(a) shows the schematic view of the nano-beam scheme. The longitudinal length of overlap region for two colliding beams is called the effective bunch length. When $\beta_{y}^{*}$ is compared with the effective bunch length, the hourglass requirement is modified by

$$
\beta_{y}^{*}>\tilde{\sigma}_{z}=\frac{\sigma_{x}^{*}}{\phi_{x}}=\frac{\sigma_{z}}{\Psi}
$$

If $\sigma_{z}$ is $6 \mathrm{~mm}$ and $\Psi$ is $20, \beta_{y}^{*}$ can be squeezed down to $300 \mu \mathrm{m}$ in principle which is much shorter than the real bunch length. In addition to reducing the hourglass effect, the vertex position along the beam axis is also restricted which provides a benefit for the primary vertex reconstruction of physics events.

\section{Crab waist scheme}

The purpose of the crab waist [2] is to reduce particles collide at the location shifted from the waist position as much as possible. The waist position is defined by the location at the smallest cross section where is the highest density. In addition to the geometrical effect, the crab waist can be expected to reduce resonance lines and a beam-tail due to beam-beam interactions [3]. The waist 
(a)

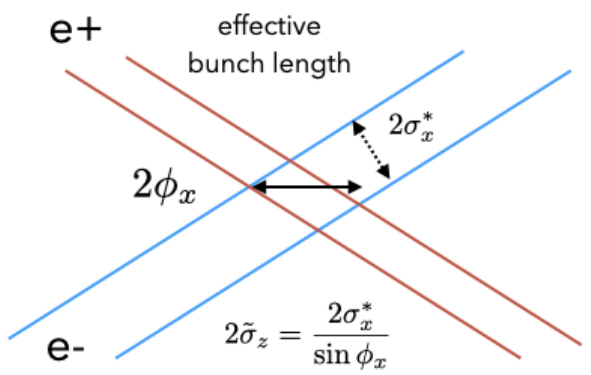

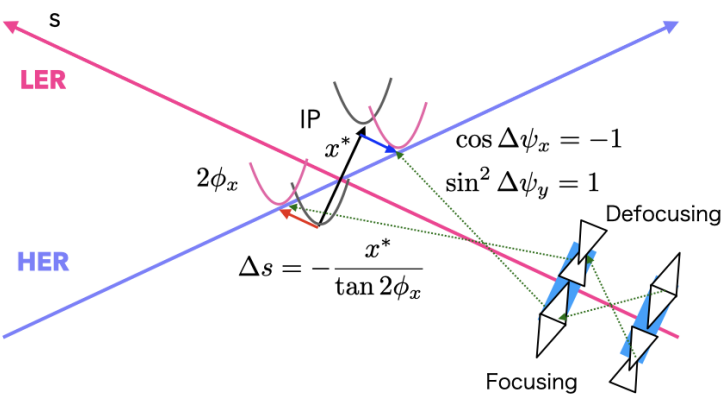

Crab waist sextupoles (-I' transfer)

(b)

Figure 1: Schematic view of nano-beam scheme (a) and crab waist scheme (b).

position can be shifted by according to the horizontal amplitude when the following Hamiltonian is created at the IP,

$$
H_{c w}=-\frac{1}{2 \tan 2 \phi_{x}} x^{*} p_{y}^{* 2} .
$$

On the other hand, a crab-waist sextupole can produce the Hamiltonian at the IP as following:

$$
H_{c w}=-\frac{K_{2}}{2} \beta_{y}^{s} \beta_{y}^{*} \sqrt{\frac{\beta_{x}^{s}}{\beta_{x}^{*}}} \cos \Delta \psi_{x} \sin ^{2} \Delta \psi_{y} x^{*} p_{y}^{* 2},
$$

where the $\Delta \psi_{x, y}$ is the phase-advance between the sextupole and the IP, $s$ indicates the sextupole. Therefore, the crab waist can be applied by choosing an appropriate phase-advance and beta functions at the sextupole. The phase-advance is adjusted to be almost $\pi$ in the horizontal and $3 \pi / 2$ in the vertical direction, respectively. The field gradient $\left(K_{2}\right)$ of the sextupole adjusts the crab-waist ratio from $0 \%$ to $100 \%$. The schematic view of the crab waist scheme in SuperKEKB is shown in figure 1(b).

The particles in the inner position at the IP are forced by the crab-waist sextupoles and the effect is defocusing according to the horizontal displacement, on the other hand, the outer particles obtain focusing effect from the crab-waist sextupoles which means different $\alpha_{y}^{*}$ function is created for each inner and outer particles. As the result of the behavior of the inner and outer particles, the waist position can be shifted along the beam line of the opposite beam due to the crossing angle between two colliding beams. In the case of SuperKEKB, the local chromaticity correction in the vertical direction is utilized to realize the crab waist [4]. There are two pairs of sextupole magnets at each side upper and downstream of the IP to confine the crab waist between them in the interaction region. The phase-advance between two sextupoles in a pair is $\pi$ and the transfer matrix is $-I^{\prime}$. When the different field gradients of $\pm \Delta K_{2}$ are applied for each sextupole in a pair, the crab waist effect can be induced with keeping the chromaticity.

\section{Achievements in 2019 run and 2020 Spring run}

Figure 2 shows the operation summary for 2019 run and 2020 Spring run. There are two long shutdowns to maintain both the accelerator and detector. It is found that the luminosity has been 
improved significantly in 2020 since 2019 run even though the maximum beam currents do not increase. The crab waist has been applied for 2020 Spring run which is expected to reduce the beam-tail and resonance lines induced by beam-beam interactions. The crab waist ratio is $80 \%$ in the LER and $40 \%$ in the HER, respectively.

The horizontal emittance was changed from $1.6 \mathrm{~nm}$ to $4 \mathrm{~nm}$ to reduce Touschek effect as much as possible. A correction of chromatic X-Y couplings at IP was also tried by using 24 rotatable sextupoles in the LER. The integrated luminosity of $74 \mathrm{fb}^{-1}$ was approximately recorded until 2020 Spring run. The peak luminosity during Belle II data acquisitions (physics run) for each operation period is shown in Table 1.

\begin{tabular}{|l|c|c|c|c|}
\hline Classification & 2018a/b & 2019a/b & 2019c & 2020a/b \\
\hline Date start & Mar. 19 & Mar. 11 & Oct. 15 & Feb. 25 \\
Date end & Jul. 17 & Jul. 1 & Dec. 12 & Jul. 1 \\
\hline Period (days) & 120 & 91 & 57 & 127 \\
\hline$\beta_{x}^{*}(\mathrm{~mm})$ & $200 / 100$ & $80 / 80$ & $80 / 60$ & $60 / 60$ \\
$\beta_{y}^{*}(\mathrm{~mm})$ & $3 / 3$ & $2 / 2$ & $1 / 1$ & $0.8 / 0.8$ \\
\hline$I_{\text {max }}(\mathrm{mA})$ & $860 / 800$ & $940 / 840$ & $880 / 700$ & $770 / 660$ \\
\hline Crab waist ratio $(\%)$ & $0 / 0$ & $0 / 0$ & $0 / 0$ & $80 / 40$ \\
\hline$L_{\text {peak }}\left(\times 10^{34} \mathrm{~cm}^{-2} \mathrm{~s}^{-1}\right)$ & 0.26 & 0.55 & 1.14 & 2.40 \\
\hline
\end{tabular}

Table 1: Operation date and period, the minimum beta function at the IP, the maximum beam currents, and peak luminosity under physics run. Left side for the LER and right for the HER to indicate $\beta_{x, y}^{*}$ and beam currents, and so on. The luminosity does not correspond to the beam currents and the beta functions at the IP.

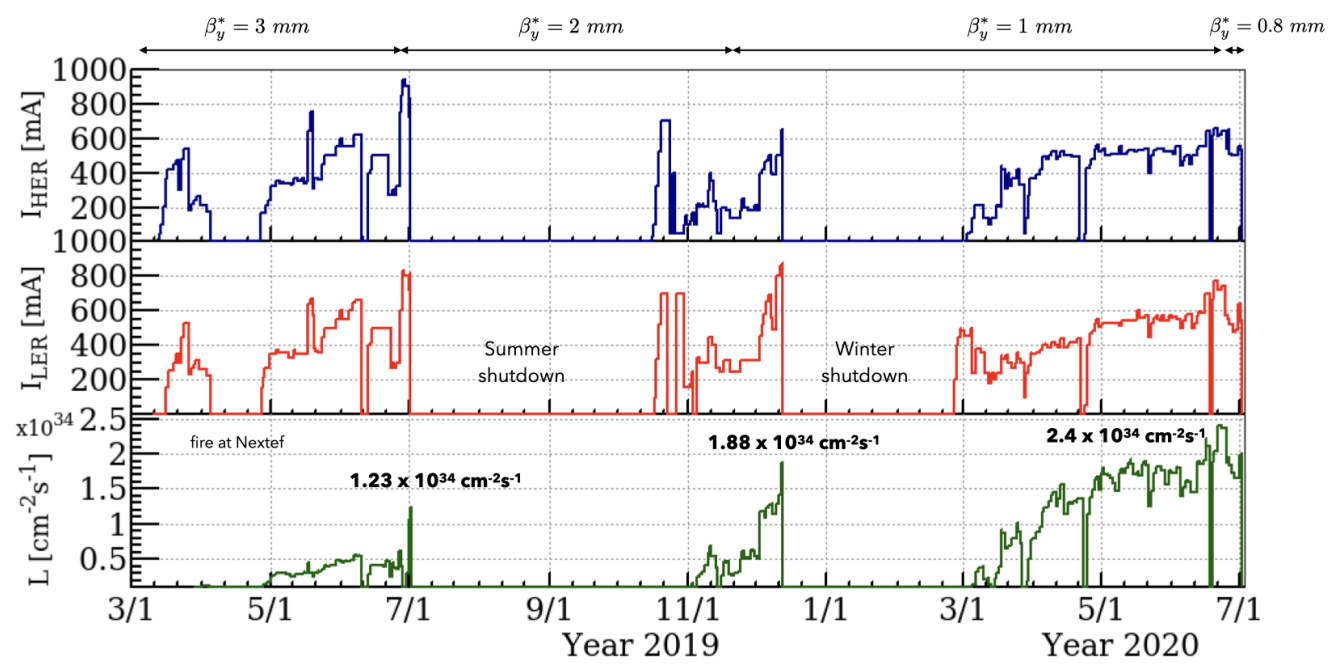

Figure 2: Operation history from 2019 run to 2020 Spring run. The beam current in the HER (top) and LER (middle), and the peak luminosity in a day without a requirement of physics run (bottom).

Figure 3(a) shows a history of $\beta_{y}^{*}$ for various colliders in the world. The $\beta_{y}^{*}$ in the future circular colliders such as FCC-ee and CEPC is 1 or $2 \mathrm{~mm}$ which is the same level or larger than the 
present SuperKEKB operation. This implies that SuperKEKB also plays a role of a challenge for the future colliders. The specific luminosity is shown in figure 3(b) for $\beta_{y}^{*}=1 \mathrm{~mm}$ and $800 \mu \mathrm{m}$. The definition of specific luminosity is

$$
L_{s p}=\frac{L}{I_{b+} I_{b-} n_{b}} \propto \frac{1}{\sqrt{\varepsilon_{y+} \beta_{y+}^{*}+\varepsilon_{y-} \beta_{y-}^{*}}},
$$

where $I_{b \pm}$ is the bunch current. If $\beta_{y}^{*}$ and $\varepsilon_{y}$ are constant, the specific luminosity should be constant. Since the specific luminosity decreases gradually as the bunch current product increases, the beam-beam blowup is observed. It is difficult to increase the bunch current product larger than $0.35 \mathrm{~mA}^{2}$ in 2019 run but it is improved in 2020 Spring run with adopting the crab waist. The specific luminosity increases as squeezing $\beta_{y}^{*}$ when the specific luminosity is between $\beta_{y}^{*}=800 \mu \mathrm{m}$ and $1 \mathrm{~mm}$. In the case of $\beta_{y}^{*}=800 \mu \mathrm{m}$, the physics run was operated at about $0.3 \mathrm{~mA}^{2}$ and a stable collision tuning could be performed. The figure shows the specific luminosity in the region larger than $0.4 \mathrm{~mA}^{2}$, however, the collision tuning is not enough due to lack of the operation time. Therefore, there is a room to improve the specific luminosity at higher bunch current product. The machine parameters achieved during 2020a/b run are shown in Table 2.

(a)

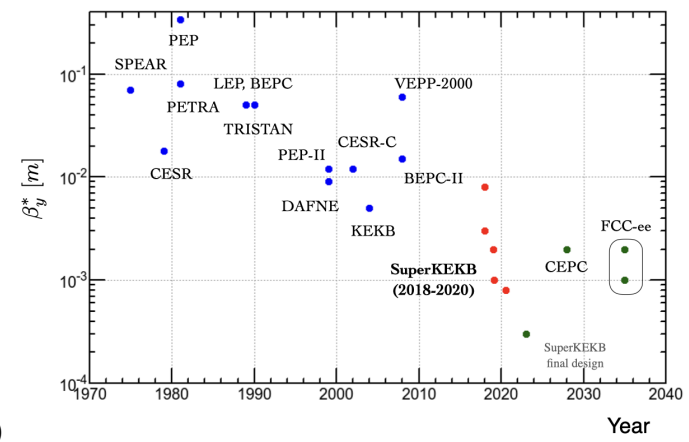

(b)

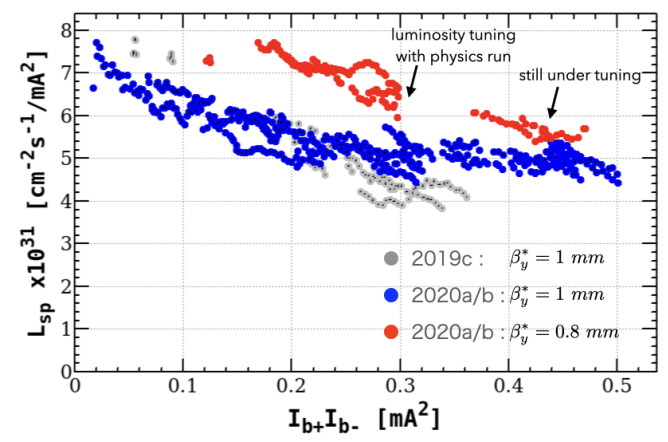

Figure 3: History of the vertical beta function (a) and specific luminosity as a function of bunch current product (b).

\section{Conclusions}

The beta function at the IP was successfully squeezed down to $800 \mu \mathrm{m}$ in the vertical direction and $60 \mathrm{~mm}$ in the horizontal direction for both the LER and HER. The vertical beam size is $224 \mathrm{~nm}$ which is the smallest value for the colliders. The smallest beam size was $700 \mathrm{~nm}$ at SLC [5] before SuperKEKB. The peak luminosity is $2.4 \times 10^{34} \mathrm{~cm}^{-2} \mathrm{~s}^{-1}$ which is the world record with $\beta_{y}^{*}=1 \mathrm{~mm}, 712 \mathrm{~mA}$ in the LER, and $607 \mathrm{~mA}$ in the HER. However, the difficulties such as extremely short lifetime and stability of operation arise significantly. The beam-related background to the Belle II detector and injector performance limit the beam current so far. The contribution of residual beam-gas background is about $50 \%$ in the LER. Therefore, further vacuum scrubbing is necessary to reduce the background. The next target for 2020 Autumn run is $4 \times 10^{34} \mathrm{~cm}^{-2} \mathrm{~s}^{-1}$ and the commissioning to improve luminosity performance has been continued. 


\begin{tabular}{lccc}
\hline & LER / HER & LER / HER & Unit \\
\hline$\varepsilon_{x}$ & $4.0 / 4.6$ & $4.0 / 4.6$ & $\mathrm{~nm}$ \\
$\beta_{x}^{*}$ & $80 / 60$ & $60 / 60$ & $\mathrm{~mm}$ \\
$\beta_{y}^{*}$ & 1 & 0.8 & $\mathrm{~mm}$ \\
$I$ & $712 / 607$ & $536 / 530$ & $\mathrm{~mA}$ \\
$n_{b}$ & 978 & 978 & \\
$I_{b}$ & $0.728 / 0.621$ & $0.548 / 0.542$ & $\mathrm{~mA}$ \\
lifetime & $760 / 1270$ & $600 / 1177$ & $\mathrm{sec}$ \\
$\sigma_{x}^{*}$ & $17.9 / 16.6$ & $15.5 / 16.6$ & $\mu \mathrm{m}$ \\
$\sigma_{y}^{*}$ & 285 & 224 & $\mathrm{~nm}$ \\
$\mathrm{CW}$ ratio & $80 / 40$ & $80 / 40$ & $\%$ \\
$\xi_{y}$ & $0.039 / 0.026$ & $0.035 / 0.020$ & \\
$L_{s p}$ & $5.4 \times 10^{31}$ & $6.9 \times 10^{31}$ & $\mathrm{~cm}^{-2} \mathrm{~s}^{-1} / \mathrm{mA}^{2}$ \\
$L$ & $2.4 \times 10^{34}$ & $2.0 \times 10^{34}$ & $\mathrm{~cm}^{-2} \mathrm{~s}^{-1}$ \\
\hline
\end{tabular}

Table 2: Machine parameters in Spring run 2020a/b.

\section{References}

[1] Y. Ohnishi et al., Accelerator Design of SuperKEKB, Prog. Theor. Exp. Phys. 2013 03A011 (2013).

[2] SuperB Conceptual Design Report, INFN/AE-07/2, SLAC-R-856, LAL 07-15, March 2007.

[3] M. Zobov et al., Crab Waist Collision Studies for $e^{+} e^{-}$Factories, arXiv:0802.2667, Feb. 2008.

[4] K. Oide et al., Beam Optics for FCC-ee Collider Ring, Proceedings of the 38th International Conference on High Energy Physics (ICHEP), Chicago, USA, Aug. 3-10, 2016.

[5] R. Assmann et al., SLC- THE END GAME, Proceedings of 7th European Conference, EPAC2000, Vienna, Austria, June 26-30, 2000. 